\title{
On the Performance of TDD Massive MIMO Systems with Pilot Contamination
}

DOI:

10.1109/VTCSpring.2017.8108329

\section{Document Version}

Accepted author manuscript

Link to publication record in Manchester Research Explorer

\section{Citation for published version (APA):}

Alkhaled, M. H. M., Alsusa, E., \& So, K. C. (2017). On the Performance of TDD Massive MIMO Systems with Pilot Contamination. In IEEE Vehicular Technology Conference Spring 2017 IEEE.

https://doi.org/10.1109/VTCSpring.2017.8108329

\section{Published in:}

IEEE Vehicular Technology Conference Spring 2017

\section{Citing this paper}

Please note that where the full-text provided on Manchester Research Explorer is the Author Accepted Manuscript or Proof version this may differ from the final Published version. If citing, it is advised that you check and use the publisher's definitive version.

\section{General rights}

Copyright and moral rights for the publications made accessible in the Research Explorer are retained by the authors and/or other copyright owners and it is a condition of accessing publications that users recognise and abide by the legal requirements associated with these rights.

\section{Takedown policy}

If you believe that this document breaches copyright please refer to the University of Manchester's Takedown Procedures [http://man.ac.uk/04Y6Bo] or contact uml.scholarlycommunications@manchester.ac.uk providing relevant details, so we can investigate your claim.

\section{OPEN ACCESS}




\title{
On the Performance of TDD Massive MIMO Systems with Pilot Contamination
}

\author{
Makram Alkhaled, Emad Alsusa, and Daniel K. C. So \\ School of Electrical and Electronic Engineering, \\ The University of Manchester, Manchester, United Kingdom \\ Email: makram.alkhaled@manchester.ac.uk; e.alsusa@manchester.ac.uk
}

\begin{abstract}
The pilot contamination problem is one of the major obstacles that limit the performance of time-division duplex (TDD) multi-cell massive multiple-input multiple-output (MIMO) systems. Pilot contamination results from the re-use of the same set of pilot sequences in the different cells of the system. In this paper, we compare between two different scenarios of pilot signals allocation with respect to the impact of pilot contamination. We derive lower bounds on the achievable rates and study the performance of both scenarios under different system settings. Our results show that although increasing the number of base station (BS) antennas improves the system performance, it does not eliminate the effect of pilot contamination. Thus, when the pilot contamination is high, it should be countered by allocating more system resources for the training phase. This can be achieved by increasing the number of pilot sequences to guarantee an orthogonal pilot sequence for each user in the system. Further, we show that the pilot sequences allocation strategy also depends on the characteristics of the communication environment: a low mobility environment has better performance when orthogonal sequences are allocated to all users while the opposite is true for a high mobility environment.
\end{abstract}

Index Terms-LSAS, massive MIMO, multicell MIMO, pilot contamination, pilot allocation.

\section{INTRODUCTION}

Massive MIMO systems have attracted a lot of attention from both academia and industry for their capabilities to provide high spectral and energy efficiency compared to conventional MIMO systems. In massive MIMO systems the BS is equipped with a very large number of service antennas (few hundreds) which are utilised to serve a smaller number of users (few tens) [1][2]. In TDD multicell massive MIMO systems, the users of each cell transmit pilot (training) sequences to their corresponding BSs. These sequences are pre-known to the BSs and orthogonal to each other. The BSs use the received pilot sequences to estimate the uplink channel matrix. Based on the estimated channel matrix, each BS builds its own detection matrix. The detection matrix is applied to the received signals vector to retrieve the uplink data symbols during the uplink phase. Moreover, based on the assumption of reciprocity between the uplink and the downlink channels, the estimated channel matrix is also utilized to build the precoding matrix which precodes the downlink data symbols during the downlink phase. Due to the scarcity of resources, the same set of pilot sequences used in one cell might be reused in the other cells. The re-use of the same set of pilot sequences results in a problem called pilot contamination.
Pilot contamination simply means that the estimations of the channels between one BS and its own users are contaminated by the channels between that BS and the users of the other cells.

Pilot contamination limits the performance of TDD massive MIMO systems in both downlink and uplink. Thus, several methods have been proposed to mitigate or reduce the effect of pilot contamination. In [3], [4] and [5], a scheme based on a time-shifted (asynchronous) pilot transmission protocol is proposed to reduce the effect of pilot contamination. The proposed scheme partitions cells into several groups $A_{1}, A_{2}, \ldots, A_{\Gamma}$ and uses a time-shifted protocol in each group. The proposed scheme ensures that there is no pilot contamination among users from different groups. Thus, it achieves the same SINR as a system with frequency reuse factor of $\Gamma$.

A multicell minimum-mean squared error (MMSE) based precoding technique is proposed in [6]. In this technique, the precoding matrix of each $\mathrm{BS}$ is designed to minimize the sum of the squared error of its own users and interference to the users in all other cells. However, this precoding method should be combined with power allocation, power control, and scheduling when the sum rate is the performance metric of interest. In [7], a multicell massive MIMO system in which collaboration between cells is permitted is considered. Based on the assumptions made in [7], an outer multi-cellular precoding referred to as Pilot Contamination Precoding (PCP) is designed to eliminate the inter-cell interference resulting from the pilot contamination problem. The main idea of PCP is that each BS linearly combines messages for users in different cells that share the same pilot sequence. The combining coefficients of the proposed PCP depend on the slow fading coefficients between the users and the BSs only. Finally, by using conventional linear precoding that is based on the estimated fast fading coefficients, each BS independently transmits its PCP-combined symbols to its users. In [8], the multipath channel model for linear antenna arrays is considered. It is shown that users with mutually non-overlapping angle of arrival (AOA) probability density functions (PDFs) hardly contaminate each other even if they use the same pilot sequences. Based on this fact, a coordinated scheme which assigns identical pilot sequences only to users of this type was proposed. The proposed scheme can significantly reduce the intercell interference and increase the uplink and the downlink SINRs. 
In this paper we consider and compare between two different scenarios for pilot sequences allocation. In the first scenario, referred to hereon as "orthogonal pilot", we assume that none of the pilot sequences assigned to any user in the system is re-used again by any other user. While in the second scenario, referred to hereon as "pilot re-use", we assume that the pilot sequences used in one cell are re-used in the other cells in the system. To evaluate the system performance in each scenario, we derive lower bounds on the achievable rates and compare between the spectral efficiency of the system in both scenarios for different system settings.

The rest of the paper is organised as follows. The system model is illustrated in Section II. Section III describes the communication scheme and presents the two scenarios for pilot sequences allocation. The lower bounds on the achievable rates and spectral efficiency for both scenarios are presented in Section IV. Numerical results are presented and discussed in Section V. Finally, conclusions are drawn in Section VI.

\section{SySTEM MODEL}

We consider the uplink of a multicell massive MIMO system with $L$ cells numbered $1,2, \ldots, L$ which are sharing the same frequency band. Each cell has one BS that is equipped with $\mathrm{M}$ service antennas to provide services to $K$ single antenna users. The users in each cell transmit data to their corresponding $\mathrm{BS}$ in the same time-frequency resources. Hence, the $M \times 1$ received signals vector at the $\mathrm{BS}$ of the $l$-th cell is given by

$$
\mathbf{y}_{l}=\sqrt{p_{u}} \sum_{i=1}^{L} \mathbf{G}_{l i} \mathbf{x}_{i}+\mathbf{n}_{l},
$$

where $p_{u}$ is the normalized transmit SNR, $\mathbf{x}_{i}$ is the $K \times 1$ vector of data symbols transmitted simultaneously by the $K$ users in the $i$-th cell, $\mathbf{n}_{l}$ is the $M \times 1$ vector of additive white zero-mean unit-variance Gaussian noise, and $\mathbf{G}_{l i}$ is the $M \times K$ channel matrix between the $K$ users in the $i$-th cell and the $M$ antennas of the $l$-th BS. The channel matrix $\mathbf{G}_{l i}$ models the independent fast fading, the geometrical attenuation, and the log-normal shadowing and can be represented as

$$
\mathbf{G}_{l i}=\mathbf{H}_{l i} \mathbf{D}_{l i}^{1 / 2}
$$

where $\mathbf{H}_{l i}$ is the $M \times K$ matrix of fast fading coefficients between the $K$ users of the $i$-th cell and the $M$ antennas of the $l$-th BS, i.e., $\left[\mathbf{H}_{l i}\right]_{m k}=h_{\text {limk }}$ is the fast fading coefficient between the $k$-th user in the $i$-th cell and the $m$ th antenna of the $l$-th BS. The elements of $\mathbf{H}_{l i}$ matrix are assumed to be independent and identically distributed (i.i.d) zero-mean, circularly-symmetric complex Gaussian $\mathcal{C N}(0,1)$ random variables and known to nobody. The matrix $\mathbf{D}_{l i}$ is a diagonal matrix of size $K \times K$, where $\left[\mathbf{D}_{l i}\right]_{k k}=\beta_{l i k}$ represents the large scale fading coefficient between the $k$-th user in the $i$-th cell and the $l$-th BS. We assume that the large scale fading coefficients are constant over many coherence intervals, independent over the antenna index $m$, and known to everybody. We further assume that all the direct gains are equal to 1 and all the cross gains are equal to $\alpha$, i.e., $\beta_{l l k}=1$ and $\beta_{l i k}=\alpha \forall i \neq l, k=1, \ldots, K$.

\section{COMmunication Scheme}

The communication scheme for the system considered in this paper consists of two phases: training and channel estimation phase, and uplink data transmission phase. Next, we discuss these two phases in more details.

\section{A. Uplink Training and Channel Estimation}

During the channel estimation process, we assume that an interval of length $\tau$ symbols is used for sending $\tau$ pilot symbols. This interval must be shorter than the channel's coherence time $T$. We further assume that all users in all cells transmit their pilot sequences simultaneously. Based on the received pilot sequences, the BSs estimate the uplink channel state information (CSI) and use these estimations to build the detection matrix.

Next, we consider two different scenarios for pilot sequences allocation, namely, pilot re-use and orthogonal pilot. For the rest of this paper, we use the subscripts $r$ and $o$ in the mathematical expressions to refer to the first and the second scenarios, respectively.

1) Orthogonal pilot: In this scenario, we assume that different and orthogonal pilot sequences are assigned for all the users in the system. To ensure orthogonality in this case, the length of the pilot sequence $\tau_{o}$ should satisfy $\tau_{o} \geq L K$. The $M \times \tau_{o}$ matrix of received pilot signals at the $l$-th BS can be represented as

$$
\mathbf{Y}_{l, o}=\sqrt{p_{o}} \sum_{i=1}^{L} \mathbf{G}_{l i} \boldsymbol{\Phi}_{i, o}+\mathbf{N}_{l, o},
$$

where $p_{o} \triangleq \tau_{o} p_{u}, \mathbf{N}_{l, o}$ is the $M \times \tau_{o}$ AWGN matrix at the l-th BS with i.i.d $\mathcal{C N}(0,1)$ elements, and $\boldsymbol{\Phi}_{i, o}$ is the $K \times \tau_{o}$ matrix of pilot sequences transmitted by the $K$ users in the $i$-th cell which satisfies

$$
\boldsymbol{\Phi}_{i, o} \boldsymbol{\Phi}_{l, o}^{\dagger}=\left\{\begin{array}{ll}
\mathbf{I}_{K} & \text { when } i=l \\
0 & \text { otherwise }
\end{array},\right.
$$

where $\mathbf{I}_{K}$ is the identity matrix of size $K \times K$.

2) Pilot re-use: Here, we assume that orthogonal pilot sequences are only assigned for the $K$ users in the $i$-th cell, and these sequences are re-used in all the other cells in the system. In other words, the $k$-th users in all cells are using the same pilot sequence. The pilot sequences used in the $i$-th cell can be represented by a $K \times \tau_{r}$ matrix $\boldsymbol{\Phi}_{i, r}\left(\tau_{r} \geq K\right)$, which satisfies $\boldsymbol{\Phi}_{i, r} \boldsymbol{\Phi}_{i, r}^{\dagger}=\mathbf{I}_{K}$. Then, the $M \times \tau_{r}$ received pilot matrix at the $l$-th $\mathrm{BS}$ is given by

$$
\mathbf{Y}_{l, r}=\sqrt{p_{r}} \sum_{i=1}^{L} \mathbf{G}_{l i} \boldsymbol{\Phi}_{i, r}+\mathbf{N}_{l, r}
$$

where $p_{r} \triangleq \tau_{r} p_{u}$, and $\mathbf{N}_{l, r}$ is the $M \times \tau_{r}$ AWGN matrix at the $l$-th BS with i.i.d $\mathcal{C N}(0,1)$ elements. 
After receiving the transmitted pilot signals, the BSs estimate their own channel matrices by utilising one of the pilotbased channel estimation methods. In this paper, we assume that MMSE channel estimation method is used. The MMSE estimate of the channel matrix $\mathbf{G}_{l i}$ given $\mathbf{Y}_{l, s}$ is given by [6]

$$
\begin{aligned}
& \hat{\mathbf{G}}_{l i, s}= \\
& \sqrt{p_{s}} \mathbf{Y}_{l, s}\left(\mathbf{I}_{\tau_{s}}+\sqrt{p_{s}} \sum_{j=1}^{L} \boldsymbol{\Phi}_{j, s}^{\dagger} \mathbf{D}_{l j} \boldsymbol{\Phi}_{j, s}\right)^{-1} \boldsymbol{\Phi}_{i, s}^{\dagger} \mathbf{D}_{l i}^{1 / 2},
\end{aligned}
$$

where $s \in\{r, o\}$.

\section{B. Uplink Data Transmission}

In this phase, the users in all cells transmit their data symbols to their corresponding BSs. Let $\mathbf{A}_{l, s}$ be the $M \times K$ linear detector matrix of the $l$-th BS which is built based on $\hat{\mathbf{G}}_{l l, s}$. In this paper, we assume that zero-forcing (ZF) linear detector is used, which is given as follows,

$$
\mathbf{A}_{l, s}=\hat{\mathbf{G}}_{l l, s}\left(\hat{\mathbf{G}}_{l l, s}^{\dagger} \hat{\mathbf{G}}_{l l, s}\right) \cdot \cdot^{-1}
$$

The $l$-th BS multiplies the received signals vector given in (1) with the decoding matrix $\mathbf{A}_{l, s}^{\dagger}$ to retrieve the transmitted data symbols as follows

$$
\mathbf{z}_{l, s}=\mathbf{A}_{l, s}^{\dagger} \mathbf{y}_{l} .
$$

From (1) and (8), the received vector in the $l$-th BS after applying a linear detector can be represented as

$$
\begin{aligned}
\mathbf{z}_{l, s} & =\sqrt{p_{u}} \mathbf{A}_{l, s}^{\dagger} \sum_{i=1}^{L} \mathbf{G}_{l i} \mathbf{x}_{i}+\mathbf{A}_{l, s}^{\dagger} \mathbf{n}_{l} \\
& =\sqrt{p_{u}} \mathbf{A}_{l, s}^{\dagger} \mathbf{G}_{l l} \mathbf{x}_{l}+\sqrt{p_{u}} \mathbf{A}_{l, s}^{\dagger} \sum_{\substack{i=1 \\
i \neq l}}^{L} \mathbf{G}_{l i} \mathbf{x}_{i}+\mathbf{A}_{l, s}^{\dagger} \mathbf{n}_{l} .
\end{aligned}
$$

\section{Achievable Rate Lower Bounds and Spectral} EFFICIENCY

\section{A. Achievable rate}

Owing to the properties of MMSE estimation, the estimation error matrix $\mathcal{E}_{s}$ which is independent of $\hat{\mathbf{G}}_{l l, s}$ is given by

$$
\mathcal{E}_{s} \triangleq\left(\mathbf{G}_{l l, s}-\hat{\mathbf{G}}_{l l, s}\right) \text {. }
$$

When $s=o$, the $j$-th column of $\mathcal{E}_{s}$ and $\hat{\mathbf{G}}_{l l, s}$ are $\mathcal{C N}\left(0, V_{j, o} \mathbf{I}_{M}\right)$ and $\mathcal{C N}\left(0,\left(1-V_{j, o}\right) \mathbf{I}_{M}\right)$, respectively [1]. While, the $j$-th column of $\mathcal{E}_{s}$ and $\hat{\mathbf{G}}_{l l, s}$ are $\mathcal{C N}\left(0, V_{j, r} \mathbf{I}_{M}\right)$ and $\mathcal{C N}\left(0,\left(1-V_{j, r}\right) \mathbf{I}_{M}\right)$ when $s=r$, respectively [6]. Whereas, $V_{j, o}$ and $V_{j, r}$ are given as follows

$$
\begin{gathered}
V_{j, o}=\frac{\beta_{l l j}}{p_{o} \beta_{l l j}+1} . \\
V_{j, r}=\frac{1+p_{r} \sum_{i \neq l}^{L} \beta_{l i j}}{1+p_{r} \sum_{i=1}^{L} \beta_{l i j}} .
\end{gathered}
$$

Substituting (10) in (9), the received vector at the $l$-th BS after using the linear detector can be rewritten as

$$
\begin{aligned}
\mathbf{z}_{l, s}=\sqrt{p_{u}} \mathbf{A}_{l, s}^{\dagger}\left(\hat{\mathbf{G}}_{l l, s}+\mathcal{E}_{s}\right) \mathbf{x}_{l} & \\
& +\sqrt{p_{u}} \mathbf{A}_{l, s}^{\dagger} \sum_{\substack{i=1 \\
i \neq l}}^{L} \mathbf{G}_{l i, s} \mathbf{x}_{i}+\mathbf{A}_{l, s}^{\dagger} \mathbf{n}_{l}
\end{aligned}
$$

Let $z_{l k, s}$ and $x_{i k}$ be the $k$-th elements of the $K \times 1$ vectors $\mathbf{z}_{l, s}$ and $\mathbf{x}_{i}$, respectively. Then, the signal of the $k$-th user at the $l$-the BS after applying the detection matrix can be expressed as

$$
\begin{aligned}
z_{l k, s}= & \sqrt{p_{u}} \mathbf{a}_{l k, s}^{\dagger} \hat{\mathbf{g}}_{l l k} x_{l k} \\
& +\sqrt{p_{u}} \sum_{\substack{j=1 \\
j \neq k}}^{K} \mathbf{a}_{l k, s}^{\dagger} \hat{\mathbf{g}}_{l l j} x_{l j}+\sum_{j=1}^{K} \mathbf{a}_{l k, s}^{\dagger} \boldsymbol{\varepsilon}_{j, s} x_{l j} \\
& +\sqrt{p_{u}} \sum_{\substack{i=1 \\
i \neq l}}^{L} \sum_{j=1}^{K} \mathbf{a}_{l k, s}^{\dagger} \mathbf{g}_{l i j} x_{i j}+\mathbf{a}_{l k, s}^{\dagger} \mathbf{n}_{l},
\end{aligned}
$$

where $\mathbf{a}_{l k, s}$ and $\mathbf{g}_{l l k}$ are the $k$-th columns of the matrices $\mathbf{A}_{l, s}$ and $\mathbf{G}_{l l}$, respectively. Assuming that the channel is ergodic so that each codeword spans over a large (infinite) number of realizations of the fast-fading factor of channel matrix $\mathbf{G}_{l i}$, then the ergodic achievable uplink rates of the $k$-th user in the $l$-th cell and for both scenarios are given by (15) and (16) shown at the top of the next page.

The lower bounds on the ergodic achievable for both scenarios with $\mathrm{ZF}$ can be found as follows:

1) Orthogonal Pilot: For ZF with Rayleigh fading, and $M \geq K+1$, the lower bound on the uplink achievable rate of the $k$-th user in the $l$-th cell is given by (17) shown at the top of next page.

Proof: See Appendix A.

2) Pilot Re-use: For pilot re-use scenario, we use the lower bound given in [1], which is given by (18) shown at the top of next page, where $\bar{L}=(L-1) \alpha+1$.

\section{B. Spectral Efficiency}

We define the spectral efficiency of the $l$-th cell as the sum of rates achieved by all the users of the $l$-th cell measured in bits/channel use. Thus the spectral efficiency of the $l$-th cell can be expressed as

$$
S E_{l, s}=\frac{T-\tau_{s}}{T} \sum_{k=1}^{K} R_{l k, s},
$$

where $T$ is the coherence interval of the channel measured in symbols. In (19), we can see that there is a trade-off between the length of the pilot sequence assigned for each user and the spectral efficiency. In the next section we discuss this trade-off in more details. 


$$
\begin{aligned}
& R_{l k, o}=\mathbb{E}\left\{\log _{2}\left(1+\frac{p_{u}\left|\mathbf{a}_{l k, o}^{\dagger} \hat{\mathbf{g}}_{l l k, o}\right|^{2}}{p_{u} \sum_{\substack{j=1 \\
j \neq k}}^{K}\left|\mathbf{a}_{l k, o}^{\dagger} \hat{\mathbf{g}}_{l l j, o}\right|^{2}+p_{u}\left\|\mathbf{a}_{l k, o}\right\|^{2} \sum_{j=1}^{K} \frac{\beta_{l l j}}{p_{o} \beta_{l j}+1}+p_{u} \sum_{\substack{i=1 \\
i \neq 1}}^{L} \sum_{j=1}^{K}\left|\mathbf{a}_{l k, o}^{\dagger} \mathbf{g}_{l i j}\right|^{2}+\left\|\mathbf{a}_{l k, o}\right\|^{2}}\right)\right\} . \\
& R_{l k, r}=\mathbb{E}\left\{\log _{2}\left(1+\frac{p_{u}\left|\mathbf{a}_{l k, r}^{\dagger} \hat{\mathbf{g}}_{l l k, r}\right|^{2}}{p_{u} \sum_{\substack{j=1 \\
j \neq k}}^{K}\left|\mathbf{a}_{l k, r}^{\dagger} \hat{\mathbf{g}}_{l l j, r}\right|^{2}+p_{u}\left\|\mathbf{a}_{l k, r}\right\|^{2} \sum_{j=1}^{K} \frac{1+p_{r} \sum_{i \neq l}^{L} \beta_{l i j}}{1+p_{r} \sum_{i=1}^{L} \beta_{l i j}}+p_{u} \sum_{\substack{i=1 \\
i \neq l}}^{L} \sum_{j=1}^{K}\left|\mathbf{a}_{l k, r}^{\dagger} \mathbf{g}_{l i j}\right|^{2}+\left\|\mathbf{a}_{l k, r}\right\|^{2}}\right)\right\} . \\
& \tilde{R}_{l k, o}^{Z F}=\log _{2}\left(1+\frac{p_{u}\left(1-V_{k, o}\right)(M-K)}{p_{u} K V_{k, o}+p_{u}(L-1) K \beta_{l i}+1}\right) . \\
& \tilde{R}_{l k, r}^{Z F}=\log _{2}\left(1+\frac{p_{u}^{2}(M-K) \tau_{r}}{\tau_{r} K\left(\bar{L}^{2}-\alpha \bar{L}+\alpha-1\right) p_{u}^{2}+\tau_{r} \alpha^{2}(L-1)(M-K) p_{u}^{2}+\bar{L}\left(K+\tau_{r}\right) p_{u}+1}\right) .
\end{aligned}
$$

\section{Numerical RESUlts}

In this section, we present some numerical results to validate our analysis. We consider a system with $L=7$ hexagonal cells that share the same time-frequency resources. The centre cell surrounded by other cells is considered as the target cell. We assume that the lengths of the pilot sequences used in the pilot re-use and orthogonal pilot scenarios are $\tau_{r}=K$ and $\tau_{o}=L K$, respectively. These lengths represent the minimum required pilot lengths to ensure orthogonality. We further assume that the transmit SNR of each user is $p_{u}=0 \mathrm{~dB}$, the cross gain $\alpha$ could be any value in the open interval $(0,1)$, and the coherence interval of the channel is $T=196$, unless stated otherwise [9].

Firstly, the effect of increasing the number of BS antennas on the system performance is shown. Figure 1 shows the spectral efficiency versus the number of BS antennas, with $\alpha=0.6$ and $K=10$. Notably, the impact of pilot contamination on the performance of the system is very significant. Whereas, the spectral efficiency starts to saturate as the number of BS antennas increases for the pilot re-use scenario, while it increases as the number of BS antennas increases for the orthogonal pilot scenario. This is due to the fact that for the pilot re-use scenario there is a residual interference from the users in the other cells that are using the same pilot sequences, and this interference cannot be eliminated by simply increasing the number of BS antennas.

Figure 2 shows the effect of the number of users on the spectral efficiency of the system for $\alpha=0.6, M=30$ and 100. It can be seen that for small number of users, the use of orthogonal pilot sequences in the different cells yields better spectral efficiency than re-using the same pilot sequences in all cells. In other words, allocating more resources for the training phase to avoid pilot contamination is more feasible than using these resources for data transmission. However, as the number of users increases, the spectral efficiency decreases for the

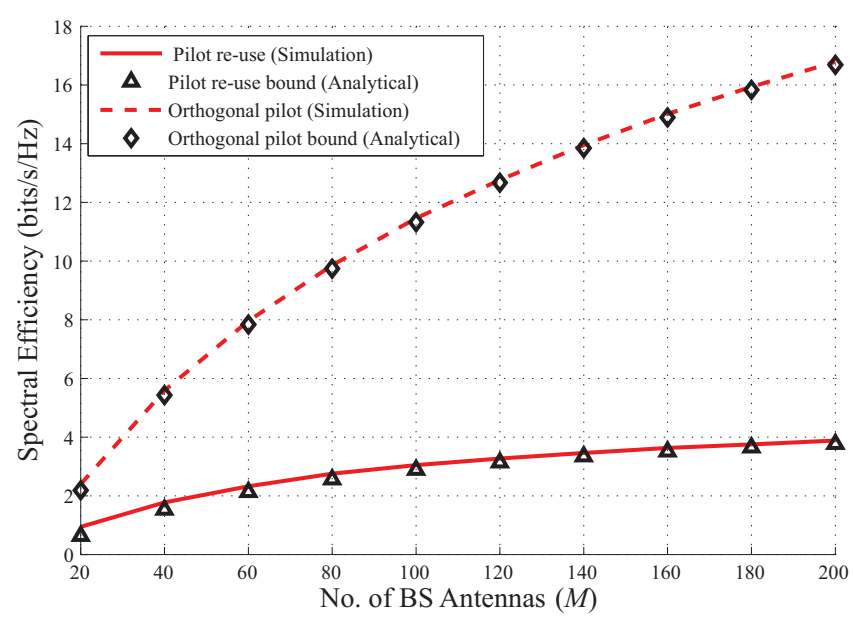

Figure 1. Spectral efficiency versus the number of BS antennas $M$, for $K=$ 10 and $\alpha=0.6$.

orthogonal pilot scenario. For large number of users (around 23 users), the spectral efficiency achieved with pilot re-use scenario becomes larger than that achieved with orthogonal pilot scenario, which indicates that it is more beneficial to assign more resources for the data transmission rather than for training. Further, Figure 2 shows that increasing the number of BS antennas results in increasing the optimum number of users that can be served simultaneously for both scenarios. For instance, the optimum number of users when $M=30$ for the orthogonal pilot scenario is 6 , which increases to 8 if $M$ is increased to 100 . While for the pilot re-use scenario, the optimum number of users is 10 which increases to 22 if $M$ is increased from 30 to 100 , respectively. In other words, the larger the number of BS antennas, the more users that can be served simultaneously.

Figure 3 depicts the spectral efficiency versus the cross gain 


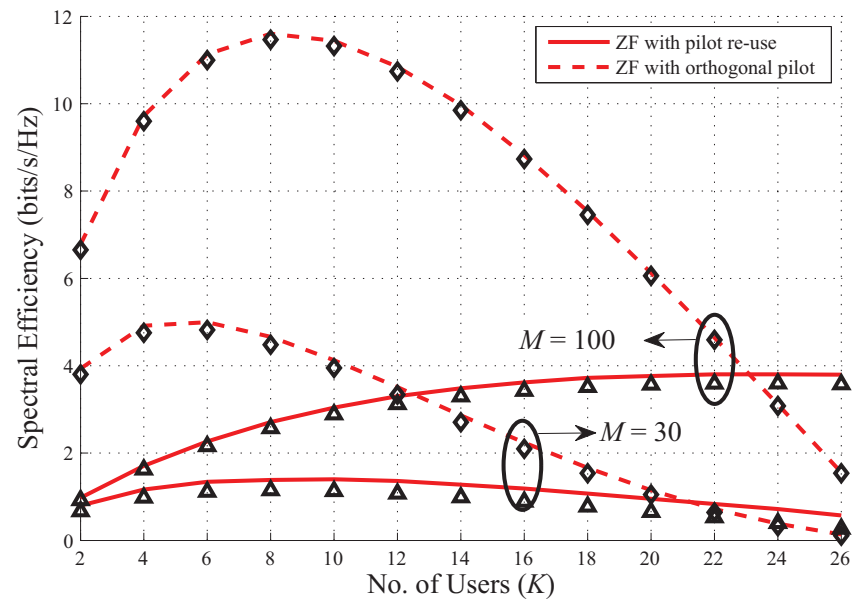

Figure 2. Lower bound on the spectral efficiency versus the number of user $K$, for $M=100$ and 30 .

$\alpha$, for $K=15$ and $M=60$. Clearly, as the cross gain value increases, the system performance degrades dramatically for the pilot re-use scenario, especially when the cross gain value becomes close to the direct gain value. This is due to the fact that as the cross gain value increases, the contamination in the channel estimation increases as well, which results in a higher inter-cell interference. On the other hand, the effect of increasing the cross gain value is less significant on the system's performance for the orthogonal pilot scenario compared to the pilot re-use scenario because there is no pilot contamination in the orthogonal pilot scenario. However, there is still inter-cell interference coming from the users in the other cells which increases with the increase in the cross gain value. Furthermore, we can notice that when the cross gain value is small compared to the direct gain value, the system's performance with pilot re-use scenario is better than its performance with orthogonal pilot scenario. However, as the cross gain value increases, the opposite holds true. This indicates that when the inter-cell interference resulting from pilot contamination is high, then it is more beneficial to utilize more system's resources to eliminate this interference (by using longer pilot sequences) rather than using these resources for transmitting data.

Finally, the spectral efficiency versus the length of the coherence interval $T$, for $\alpha=0.3, M=100$, and $K=20$ is shown in Figure 4. One can notice that, for short coherence intervals (high mobility environment), it is better to re-use the same pilot sequences in the different cells as this will reduce the length of the pilot sequences used, which means sustaining more resources for data transmission. However, for moderate and large coherence intervals, the use of orthogonal pilot sequences becomes more feasible as it results in a better performance.

\section{CONCLUSIONS}

In this paper, we have considered two different scenarios for pilot signals allocation in TDD multicell massive MIMO

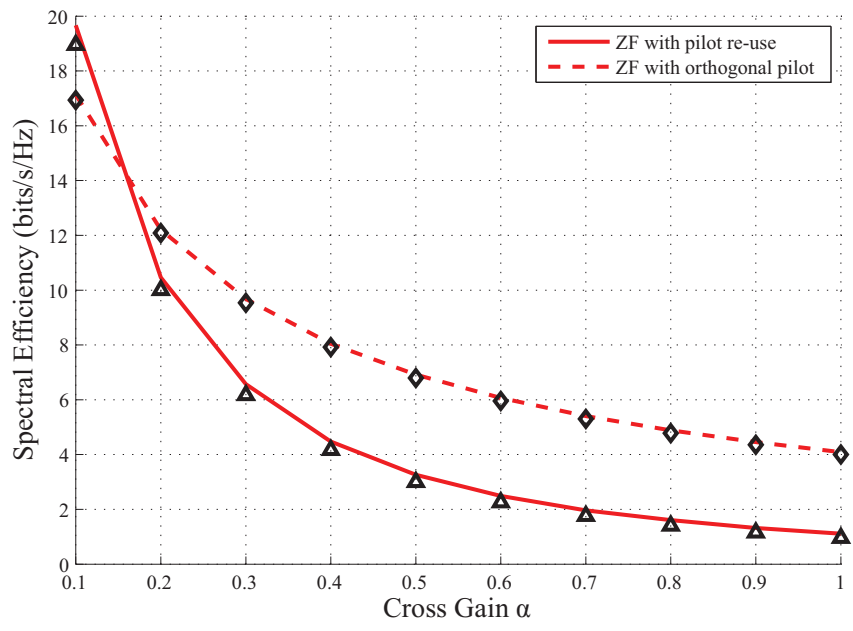

Figure 3. Spectral efficiency versus the cross value, for $M=60$ and $K=15$.

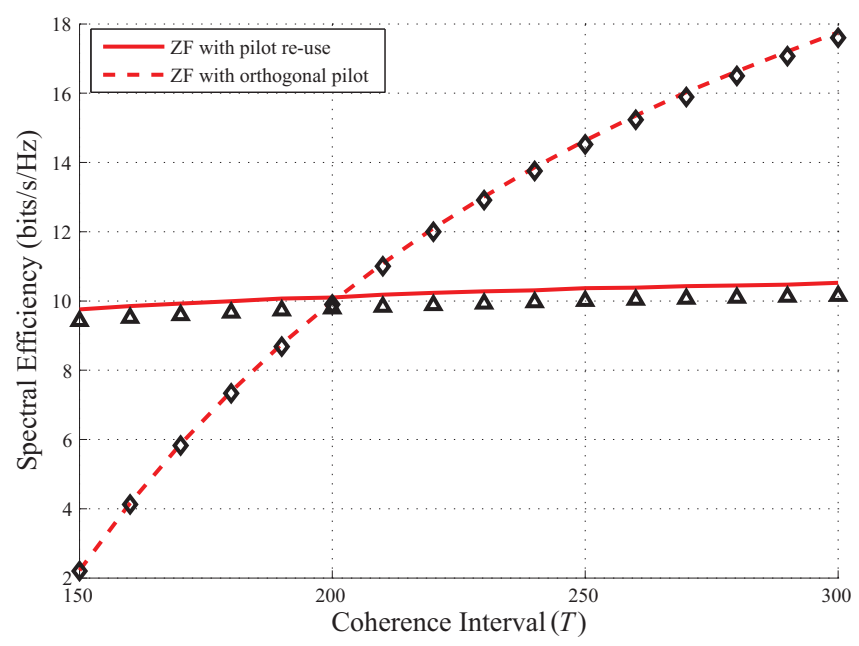

Figure 4. Spectral efficiency versus the coherence interval, for $\alpha=0.3$, $M=100$, and $K=20$.

systems. In the first scenario, we assume that the same set of pilot sequences is used in all system's cells, which results in pilot contamination during the channel estimation process. While in the second scenario, we assume that an orthogonal pilot sequence is allocated for each user in the system to mitigate the pilot contamination problem. We compare the system performance for both scenarios in terms of spectral efficiency. Our numerical results have shown that increasing the number of BS antennas can not resolve the problem of pilot contamination. Thus, when the effect of pilot contamination is very significant, orthogonal pilot sequences should be used because this results in better system performance despite consuming more system resources. Furthermore, we have shown that when the coherence time of the channel is large (limited mobility environment), then the use of orthogonal pilot sequences yields better system performance compared to the pilot re-use case, and the opposite holds true when the 


$$
\begin{aligned}
& R_{l k, o}^{Z F}=\mathbb{E}\left\{\log _{2}\left(1+\frac{p_{u}}{p_{u}\left\|\mathbf{a}_{l k, o}\right\|^{2} \sum_{j=1}^{K} \frac{\beta_{l l j}}{p_{o} \beta_{l l j}+1}+p_{u} \sum_{\substack{i=1 \\
i \neq l} \sum_{j=1}^{K}\left|\mathbf{a}_{l k, o}^{\dagger} \mathbf{g}_{l i j}\right|^{2}+\left\|\mathbf{a}_{l k, o}\right\|^{2}}}\right)\right. \\
& R_{l k, o}^{Z F} \geq \tilde{R}_{l k, o}^{Z F}=\log _{2}\left(1+\mathbb{E}\left\{\frac{\frac{p_{u}}{\left\|\mathbf{a}_{l k, o}\right\|^{2}}}{p_{u} \sum_{j=1}^{K} \frac{\beta_{l l j}}{p_{o} \beta_{l l j}+1}+p_{u} \sum_{\substack{i=1 \\
i \neq l}}^{L} \sum_{j=1}^{K} \frac{\left|\mathbf{a}_{l k, o}^{\dagger} \mathbf{g}_{l i j}\right|^{2}}{\left\|\mathbf{a}_{l k, o}\right\|^{2}}+1}\right\}\right) . \\
&=\mathbb{E}\left\{\frac{p_{u}}{\left\|\mathbf{a}_{l k, o}\right\|^{2}}\right\}\left(\frac{1}{p_{u} K V_{k, o}+p_{u} \sum_{\substack{i=1 \\
i \neq l}}^{L} \sum_{j=1}^{K} \mathbb{E}\left\{\frac{\left|\mathbf{a}_{k, o}^{\dagger} \mathbf{g}_{l i j}\right|^{2}}{\left\|\mathbf{a}_{l k, o}\right\|^{2}}\right\}+1}\right) .
\end{aligned}
$$

coherence interval is small.

\section{APPENDIX}

\section{A. ZF Lower Bound with Orthogonal Pilot Allocation}

By substituting $\mathbf{a}_{l k, o}=\hat{\mathrm{g}}_{l l k, o}\left(\hat{\mathrm{g}}_{l l k, o}^{\dagger} \hat{\mathrm{g}}_{l l k, o}\right)^{-1}$ in (15), we get (20) shown at the top of this page, and by using Jensen's inequality, we can write the lower bound as (21). The expectation in (21) can be written as (22). Then, by condition on $\mathbf{a}_{l k, o}$ we can find

$$
\mathbb{E}\left\{\frac{\left|\mathbf{a}_{l k, o}^{\dagger} \mathbf{g}_{l i j}\right|^{2}}{\left\|\mathbf{a}_{l k, o}\right\|^{2}}\right\}=\beta_{l i j} .
$$

It is known that $\left\|\mathbf{a}_{l k, o}\right\|^{2}=\left[\left(G^{H} G\right)^{-1}\right]_{k k}[10]$. Therefore,

$$
\begin{aligned}
\mathbb{E}\left\{\left[\left(G^{H} G\right)^{-1}\right]_{k k}\right\} & =\mathbb{E}\left\{\frac{\left[\left(H^{H} H\right)^{-1}\right]_{k k}}{\left(1-V_{k, o}\right)}\right\}, \\
& =\mathbb{E}\left\{\frac{\operatorname{tr}\left[\left(H^{H} H\right)^{-1}\right]}{K\left(1-V_{k, o}\right)}\right\}, \\
& =\frac{1}{(M-K)\left(1-V_{k, o}\right)} .
\end{aligned}
$$

Finally, substituting (23) and (24) into (22) and (21) we get (17).

\section{REFERENCES}

[1] H. Q. Ngo, E. Larsson, and T. Marzetta, "Energy and spectral efficiency of very large multiuser MIMO systems," IEEE Trans. Commun., vol. 61, no. 4, pp. 1436-1449, Apr. 2013.

[2] E. Larsson, O. Edfors, F. Tufvesson, and T. Marzetta, "Massive MIMO for next generation wireless systems," IEEE Commun. Mag., vol. 52, no. 2, pp. 186-195, Feb. 2014.

[3] F. Fernandes, A Ashikhmin, and T Marzetta, "Inter-cell interference in noncooperative tdd large scale antenna systems," IEEE J. Sel. Areas Commun., vol. 31, no. 2, pp. 192-201, February 2013.

[4] K. Appaiah, A. Ashikhmin, and T. Marzetta, "Pilot contamination reduction in multi-user tdd systems," in Proc. IEEE Int. Conf. Commun. (ICC), May 2010, pp. 1-5.

[5] F. Fernandes, A. Ashikhmin, and T. Marzetta, "Interference reduction on cellular networks with large antenna arrays," in Proc. IEEE Int. Conf. Commun. (ICC), June 2012, pp. 1-5.

[6] J. Jose, A. Ashikhmin, T. Marzetta, and S. Vishwanath, "Pilot contamination and precoding in multi-cell tdd systems," IEEE Trans. Wireless Commun., vol. 10, no. 8, pp. 2640-2651, Aug. 2011.

[7] A. Ashikhmin and T. Marzetta, "Pilot contamination precoding in multicell large scale antenna systems," in Proc. IEEE Int. Symp. on Info. Theory (ISIT), July 2012, pp. 1137-1141.

[8] H. Yin, D. Gesbert, M. Filippou, and Y. Liu, "A coordinated approach to channel estimation in large-scale multiple-antenna systems," IEEE J. Sel. Areas Commun., vol. 31, pp. 264-273, February 2013.

[9] T. Marzetta, "Noncooperative cellular wireless with unlimited numbers of base station antennas," IEEE Trans. Wireless Commun., vol. 9, no. 11, pp. 3590-3600, Nov. 2010.

[10] A. M. Tulino and S. Verdu, Random matrix theory and wireless communications. 40 West 20th Street, New York, USA: Now Publishers Inc., 2004. 